\title{
Decisión de Comprar o Alquilar Activos Fijos
}

\begin{abstract}
RESUMEN
Las organizaciones requieren de activos para producir $\mathrm{y} / 0$ vender sus productos en condiciones competitivas, generando valor para sus accionistas, trabajadores y la sociedad en general. Dichos activos están constituidos, principalmente, por dinero en efectivo y por activos fijos. Los activos fijos, constituidos por inmuebles, maquinaria y equipos, conforme pasa el tiempo, se desgastan y requieren ser repuestos para asegurar la calidad de los productos. El tema de decisión es la selección entre comprar los activos fijos o contratar con el proveedor el alquiler de estos activos. En el artículo se plantea una metodología de análisis de las alternativas de comprar 0 alquilar activos, con la finalidad de optimizar el valor de una empresa en marcha, utilizando criterios financieros de rentabilidad y liquidez.
\end{abstract}

Palabras clave: Activos fijos, ahorro fiscal, rentabilidad, liquidez.

Decision to Buy or Rent Fixed Assets

\section{ABSTRACT}

Organizations require assets to produce and/or sell their products under competitive conditions, generating value for its shareholders, employees and society in general. These assets consist primarily of cash and fixed assets. Fixed assets consisting of buildings, machinery and equipment, over time, become worn and require replacement to ensure product quality. The theme of choice is the choice between buying fixed assets or contract hire provider of these assets. The article presents a methodology for analyzing alternatives to buy or lease assets for the purpose of optimizing the value of a going concern, using criteria of financial profitability and liquidity.

Key words: Fixed assets, tax savings, profitability and liquidity.

\section{INTRODUCCIÓN}

Toda empresa requiere de activos para desarrollar sus actividades comerciales. La mixtura de los activos depende del giro del negocio y de los planes de desarrollo empresarial. Lo usual es disponer de fondos líquidos y de infraestructura operativa. Los fondos líquidos constituyen el activo corriente, el que se registra en el balance general de una organización a una fecha determinada, y están representados por efectivo (caja y bancos), inventarios, cuentas por cobrar y por todo derecho de la empresa que pueda hacerlo efectivo (líquido en términos de disponibilidad de fondos) en los próximos doce meses. En cambio, la infraestructura operativa está compuesta por inmuebles, maquinaria e equipos -comúnmente llamados activos fijos-, por activos intangibles y por inversiones en acciones de otras empresas, los que se contabilizan como activo no corriente. Los activos fijos permiten que la empresa desarrolle el ciclo de generación de valor, es decir, comprar insumos, procesarlos, distribuir y comercializar los productos resultantes.

Contabilizar los activos fijos en el balance general de la empresa, implica que estos han sido adquiridos (comprados) utilizando algún esquema de financiamiento que implique la mezcla de deuda y capital propio de los accionistas, los que están registrados en dicho balance como pasivo y patrimonio neto, respectivamente. El rendimiento o retorno de la inversión en activos deberá ser tal que compense el costo de capital de la empresa, expresado este costo como el promedio ponderado del costo de las fuentes de financiamiento (medido en términos de tasa de interés). Sin embargo, una forma alternativa comercial de contar con activos fijos es contratando en alquiler su uso, según las condiciones del mercado de proveedores de dichos bienes de capital.

Aquí el dilema para los directivos de la empresa es adoptar la decisión más conveniente entre comprar o alquilar los activos fijos requeridos en su proceso productivo, de modo que la decisión contribuya a mejorar el valor de la organización empresarial. En el análisis, además de otros factores, se deben contemplar dos criterios financieros, que son la rentabilidad de los fondos invertidos y la liquidez de los recursos disponibles para atender oportunamente las obligaciones de la empresa.

De la revisión de los estados financieros de las empresas, cuyas acciones representativas del capital social cotizan en la Bolsa de Valores de Lima, se aprecia que, según las condiciones del mercado que atienden, estas utilizan una combinación de alternativas de comprar y alquilar ac-

(1) Ingeniero Industrial, UNI; y Magíster en Administración, Universidad ESAN. Profesor Principal de la Facultad de Ingeniería Industrial de la UNMSM. E-mail: nestor_sj@hotmail.com 
tivos fijos. En la decisión se habría tenido en cuenta que, en la etapa de operación del negocio (luego de la inversión), los activos fijos comprados son depreciados contablemente, afectando directamente las utilidades (en el estado de ganancias y pérdidas se incluye como costo a la depreciación), lo que de manera indirecta influye en la mayor disponibilidad de caja (menos pago del impuesto a la renta); en cambio, si los activos fijos son tomados en alquiler, los gastos de operación afectan directamente al estado de ganancias y pérdidas (gastos de alquiler) e influyen en el flujo de caja, en forma directa, al registrar como egreso el pago de gastos de alquiler, y de manera indirecta, se aprecia por la mejora en el saldo del flujo de caja por efecto del menor pago de impuesto a la renta, el que se traduce en ahorro para la empresa.

El objetivo del presente estudio es analizar el modelo financiero aplicable en la toma de decisiones de una empresa, en cuanto a comprar o alquilar activos fijos, utilizando como criterios la rentabilidad y la liquidez. Dicho modelo, complementado con otros aspectos no financieros, contribuirá a que la empresa adopte decisiones integrales, en su propósito de optimizar el valor de sus acciones; será un instrumento de gestión de suma importancia en toda organización empresarial que esté afecto al régimen general del impuesto a la renta.

El análisis que se desarrolla a continuación toma como referencia a una empresa en marcha (en operación o en funcionamiento), que genera utilidades $y$, por ende, está afecta al pago de impuesto a la renta, previa deducción de la participación de utilidades a favor de sus trabajadores, según la normatividad legal vigente. Dicha empresa se encuentra en la necesidad de reponer sus activos fijos, para mantener su capacidad operativa en cuanto a cantidad y calidad de sus productos, o cuenta con planes de crecimiento para atender la mayor demanda del mercado actual y/o diversificar su cartera de clientes.

\section{ESTRATEGIA DE DESARROLLO EMPRESARIAL}

Las empresas desarrollan sus actividades operativas (productivas, administrativas y comerciales) en concordancia con el plan estratégico, en donde se contemplan la visión y misión de la organización, así como los objetivos estratégicos y los planes de acción que deben ejecutar las diversas áreas.

El plan estratégico es un instrumento de gestión de mediano y largo plazo, que requiere implementarse y ejecutarse con el compromiso de todos los inte- grantes de la empresa, y evaluarse periódicamente y de manera objetiva los resultados logrados. Las áreas de la empresa deben desarrollar sus actividades operativas -el día a día- siguiendo el esquema general (plan estratégico) diseñado por la organización para los próximos meses y años. El área de gestión financiera, cuya responsabilidad básica es administrar eficientemente los recursos financieros (fondos) de la empresa, adquiere un rol de suma trascendencia al facilitar información e instrumental necesario para analizar técnicamente las alternativas que se presenten en la empresa, para llevar a la realidad, oportuna y adecuadamente, los planes de acción de la empresa.

Los resultados de la evolución de una empresa, según los planes de acción que adopte, en el contexto que le ofrece su entorno o medio ambiente empresarial, se reflejan en sus estados financieros, de donde se pueden obtener indicadores de liquidez, solvencia, gestión y rentabilidad. Pero el efecto global de las decisiones se reflejará en el valor de las acciones representativas del capital social de la empresa, el cual se traducirá en un beneficio real y/o potencial para sus accionistas, sus trabajadores, el Estado y la sociedad en su conjunto. Como el desarrollo estratégico de la empresa debe visualizarse en el mediano y largo plazo, es decir, en los próximos cinco a diez años, en promedio, es usual que en la empresa se presenten conflictos entre el enfoque de mediano y largo plazo, y la gestión de corto plazo, la que se traduce en la necesidad de contar con resultados inmediatos.

Así las empresas, periódicamente identifican, analizan y seleccionan alternativas de desarrollo organizacional. Entre las alternativas básicas están las de reponer o no los activos fijos que ya cumplieron con su vida económica, ampliar o no la capacidad instalada (máxima producción posible bajo determinadas condiciones) y si la ampliación de la capacidad debe ser en forma concéntrica (en negocios relacionados) o de manera conglomerada (en diversos negocios). La disponibilidad de recursos financieros, sea de fondos propios y de fondos de terceros, es fundamental para llevar a la práctica la mejor alternativa de decisión que se adopte.

Una organización empresarial no debe conformarse con los resultados económicos y financieros que viene obteniendo, aunque sean estos muy satisfactorios a los intereses de sus dueños (accionistas); sino que debe, permanentemente, a través de sus dirigentes y trabajadores en general, visualizar el escenario probable que se podría presentar para el futuro del negocio. Aquí, como ayuda para la toma de 


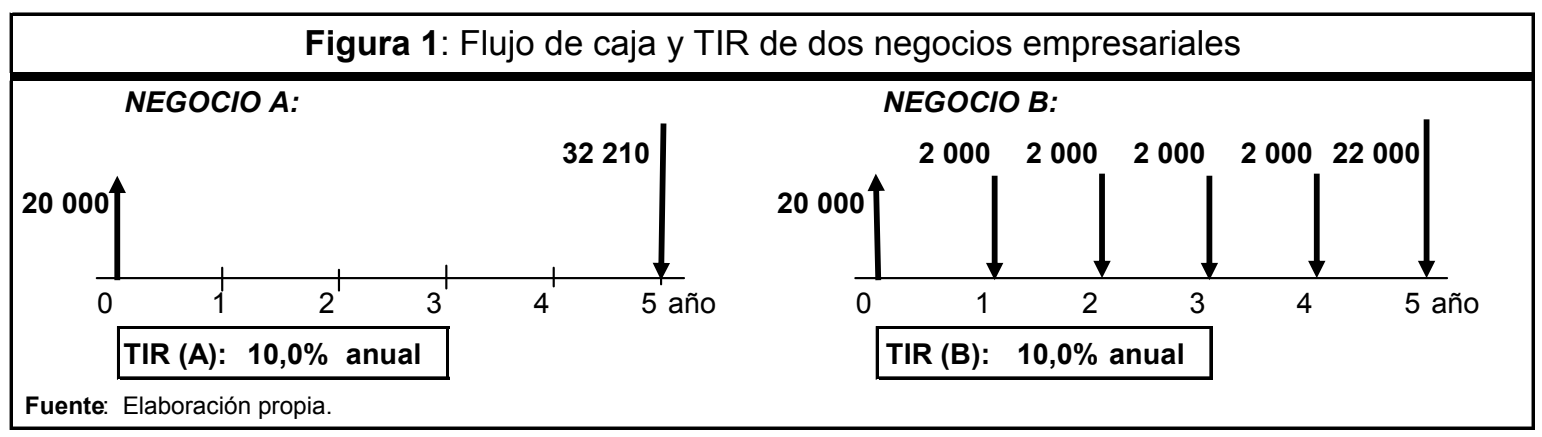

decisiones, la teoría nos ofrece modelos financieros que permiten estimar el efecto de las decisiones en el valor de las acciones de la empresa, que merecen ser puestos en práctica, según la naturaleza de cada organización.

\section{CRITERIOS FINANCIEROS DE RENTABILIDAD Y LIQUIDEZ}

El modelo financiero básico para el análisis de alternativas empresariales, considera como criterios de decisión a los índices de rentabilidad, como el Valor Presente Neto (VPN) y la Tasa Interna de Retorno (TIR), principalmente. La rentabilidad de una alternativa, medida en función al flujo de caja del negocio y el rendimiento mínimo esperado por el accionista (expresado como un costo de oportunidad), es un instrumento de análisis de mediano y largo plazo.

Para el flujo de caja de un determinado negocio, es posible hallar la TIR. Si el valor de la TIR, que es una tasa de interés, supera al costo de oportunidad del accionista, se concluye que financieramente, el negocio es rentable. Sin embargo, es importante tener cuidado en la interpretación de la TIR. En la figura 1 se tiene que dos flujos de caja, correspondientes a diferentes negocios, tienen la misma TIR.

Según la indicada figura, tanto para el Negocio A como para el Negocio $B$, se proyectan rendimientos de $10 \%$ anual (rentabilidad), con lo cual ambos negocios serían atractivos para los inversionistas (accionista) cuyo costo de oportunidad sea $10 \%$ anual. Pero, en el Negocio A, el rendimiento anual de la inversión es $0 \%$ y toda la ganancia -así como la recuperación del capital- recién se da en el año cinco; en cambio con el Negocio B, cada año se obtiene el rendimiento esperado (10\%) y la inversión inicial se recupera también en el último año de la proyección. De esta manera, se puede apreciar que el Negocio A tiene un mayor riesgo (probabilidad de no recuperar el capital invertido y obtener el rendimiento esperado) que el Negocio $B$. La rentabilidad es un concepto financiero de mediano y largo plazo, que en términos de la TIR, se expresa como un rendimiento promedio anual.

En cuanto al VPN, para un costo de oportunidad menor a $10 \%$ anual, el Negocio A resulta con una rentabilidad monetaria mayor que la del Negocio B. Por ejemplo, para un costo de oportunidad de $8 \%$ anual, el VPN del Negocio A resulta S/.1922 y el VPN del Negocio B es S/.1597, como valores equivalentes en el año cero (momento de la inversión), valores que representan una ganancia neta para el accionista, luego de recuperar su capital (S/.20 000) y obtener con este capital un rendimiento anual promedio de $8 \%$ anual. Nótese que en términos de la TIR, ambas alternativas de negocios son equivalentes (rinden $10 \%$ anual).

De otro lado, el éxito en el mediano y largo plazo será tangible, siempre que las premisas utilizadas en el análisis se encuentren dentro del rango previsto, y las acciones de corto plazo se orienten al logro de dicho resultado. $Y$ así se tiene que conjuntamente con la rentabilidad, es importante, desde el punto de vista financiero, contar con liquidez suficiente para cubrir los costos corrientes (de cada ejercicio) y que el negocio, para mitigar el riesgo de toda inversión, contribuya con sus accionistas, mediante una política adecuada y oportuna de pago de dividendos en efectivo.

\section{MODELO FINANCIERO GENERAL}

El modelo general de análisis financiero de alternativas de decisión, entre comprar o alquilar activos fijos, implica proyectar los resultados económicos y financieros de la empresa considerando -por separado- el efecto de cada alternativa en la empresa, para seleccionar aquella alternativa que financieramente contribuya a aumentar el valor de las acciones de la organización.

De esta manera, bajo determinadas premisas, se proyectan el estado de ganancias y pérdidas y el flujo de caja de la empresa, para cada alternativa 


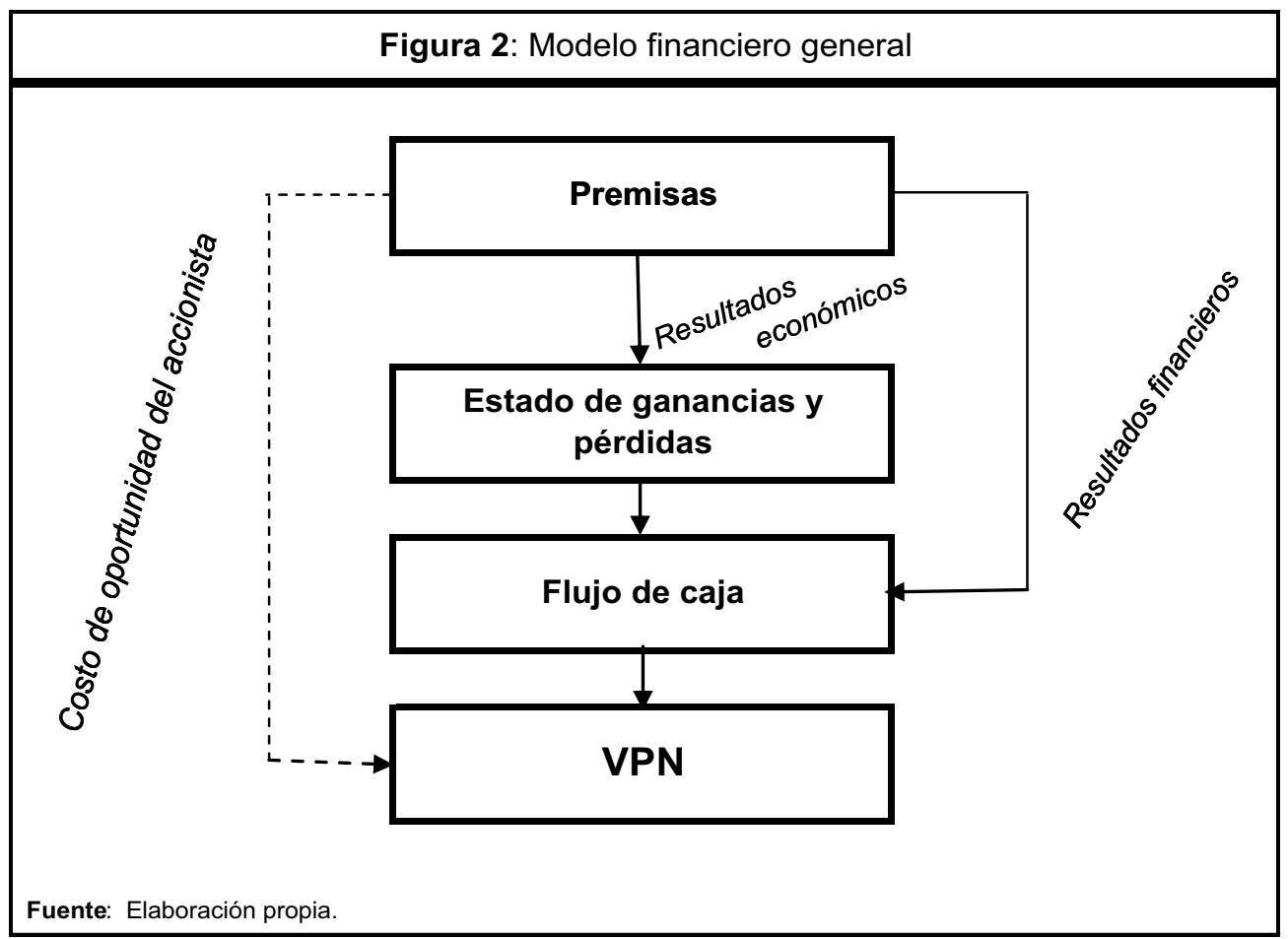

considerada en el análisis. En el estado de ganancias y pérdidas, se estiman el impuesto a la renta y la participación de los trabajadores, según la legislación vigente, los que forman parte de los egresos del flujo de caja. Con el saldo neto del flujo de caja (ingresos menos egresos de efectivo) y el costo de oportunidad del accionista (tasa mínima de retorno esperada) se determina el VPN. La alternativa con el mayor resultado del VPN se selecciona como la mejor alternativa. El modelo general de análisis se muestra en la figura 2.

Para ilustrar la aplicación, vamos a asumir que una empresa en marcha requiere de activos fijos, para realizar actividades comerciales que le permitan obtener ganancias netas en efectivo (antes de depreciación y de gastos de alquiler, y otros rubros relacionados a dichos activos), en los niveles que se detallan en el cua- dro 1, para un horizonte de proyección de cinco años; donde la decisión se debe adoptar en el año cero, de modo que los periodos operativos corresponden a los años uno al cinco. Para simplificar el análisis, se puede considerar que el valor residual (valor de salvamento o valor de rescate de los activos fijos) se hace efectivo en el último año de proyección, por un monto equivalente a su valor neto en libros contables al cierre del quinto año de proyección (inversión menos depreciación acumulada).

En el indicado cuadro también se nuestra el costo de adquirir los activos fijos (inversión), los que se deprecian para fines contables a la tasa de $12,5 \%$ anual (depreciación uniforme en ocho años). Asimismo, se tiene el costo del alquiler anual de los activos fijos (pago por año adelantado y una garantía -pago único recuperable- equivalente al $25 \%$ del

\begin{tabular}{|c|c|c|c|c|c|c|}
\hline Concepto & 0 & 1 & 2 & 3 & 4 & 5 \\
\hline Ganancias netas en efectivo $\left({ }^{*}\right)$ & & 24000 & 30000 & 36000 & 36000 & 36000 \\
\hline \multicolumn{7}{|l|}{ Alternativa de comprar activos fijos: } \\
\hline Inversión & 50000 & \multirow[b]{2}{*}{4000} & \multirow[b]{2}{*}{5000} & \multirow[b]{2}{*}{6000} & \multirow[b]{2}{*}{6000} & \multirow[b]{2}{*}{6000} \\
\hline Costo anual de operación y mantenimiento & & & & & & \\
\hline \multicolumn{7}{|l|}{ Alternativa de alquilar activos fijos: } \\
\hline Garantía del alquiler $\quad 25 \%$ & 2750 & \multirow{3}{*}{$\begin{array}{r}11000 \\
4000\end{array}$} & \multirow{3}{*}{$\begin{array}{r}11000 \\
5000\end{array}$} & \multirow{3}{*}{$\begin{array}{r}11000 \\
6000\end{array}$} & \multirow{3}{*}{$\begin{array}{r}11000 \\
6000\end{array}$} & \multirow[b]{3}{*}{6000} \\
\hline Alquiler anual & 11000 & & & & & \\
\hline Costo anual de operación y mantenimiento & & & & & & \\
\hline
\end{tabular}


costo del alquiler anual). Con ambas alternativas, comprar y alquilar, los costos anuales de operación y mantenimiento son asumidos por la empresa, obligaciones que se pagan al final de cada ejercicio. Para los fines del análisis, estos valores se muestran sin IGV y se asume que todas las operaciones comerciales son al contado y no hay inventarios, lo cual es una facilidad que nos brindan los modelos financieros, por lo que en la decisión final, es imperativo contemplar otros factores de análisis.

Adicionalmente, se tiene que la empresa interesada en los activos fijos está afecta al impuesto a la renta del $30 \%$ de sus utilidades anuales, cuyo pago, para los fines del modelo financiero, se realiza en el mismo ejercicio en que es devengado. En el análisis se asume que la empresa no está afecta al pago de participación a trabajadores, la cual equivale a un porcentaje de la utilidad antes del impuesto a la renta y que para el cómputo de dicho impuesto, la referida participación se deduce como "gasto".

En el análisis se considera un costo de oportunidad de $12 \%$ anual, que es el rendimiento mínimo requerido por los accionistas de la empresa para la toma de decisiones desde el punto de vista financiero. Dicho costo representa el rendimiento de la mejor alternativa dejada de hacer (sacrificada) por los accionistas al comprometer sus recursos en la empresa, más una prima o cobertura por riesgo (variabilidad de los resultados futuros esperados del negocio desarrollado por la empresa).
De esta manera, para cada alternativa, se proyecta el estado de ganancias y pérdidas, y luego el flujo de caja, y con el saldo neto del flujo de caja de cada periodo se determina el correspondiente VPN.

\section{Alternativa Comprar}

En el estado de ganancias y pérdidas se incluye el costo de la deprecación de los activos fijos, con lo cual, la empresa pagará menos impuesto a la renta, efecto que se verá reflejado en el rubro de egresos del flujo de caja. Asimismo, en el flujo de caja, en el año cero se concretará la inversión (costo de adquisición de los activos fijos) y en el año cinco, como ingreso de caja, se tendrá el valor residual de dichos activos. Los resultados se aprecian en el cuadro 2.

\section{Alternativa Alquilar}

En este caso, en el estado de ganancias y pérdidas se incluye el costo del alquiler, en el año en que los activos fijos son utilizados por la empresa, independientemente de la oportunidad del pago; aquí no corresponde incluir la depreciación como costo, toda vez que los activos no son de propiedad de la empresa. En el flujo de caja se considera el pago del alquiler (año adelantado, es decir al final del año anterior en que los activos son utilizados), así como el pago de garantía (año cero) y su recupero (en el año cinco). En el cuadro 3, se tienen los resultados proyectados de esta alternativa.

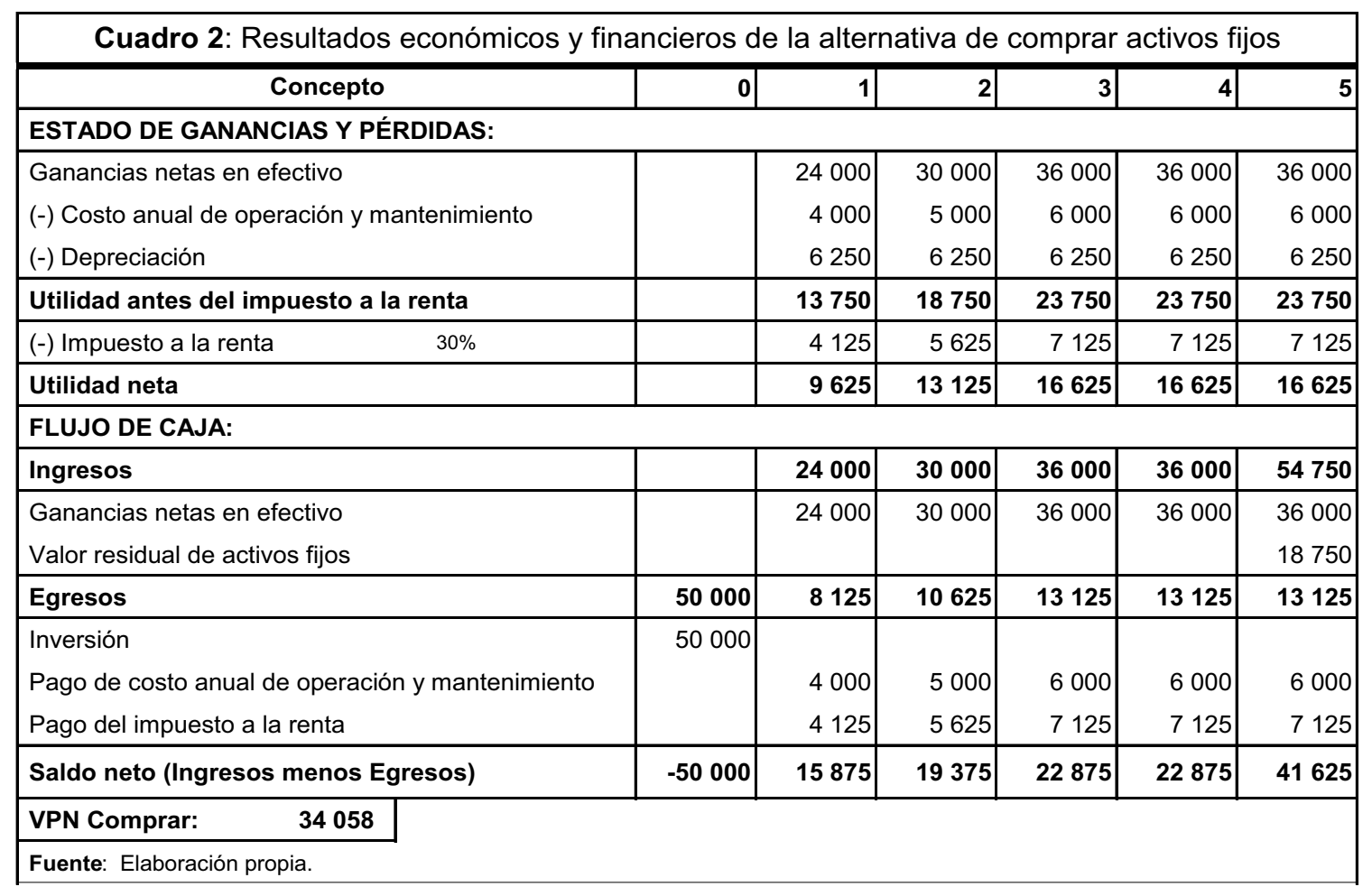




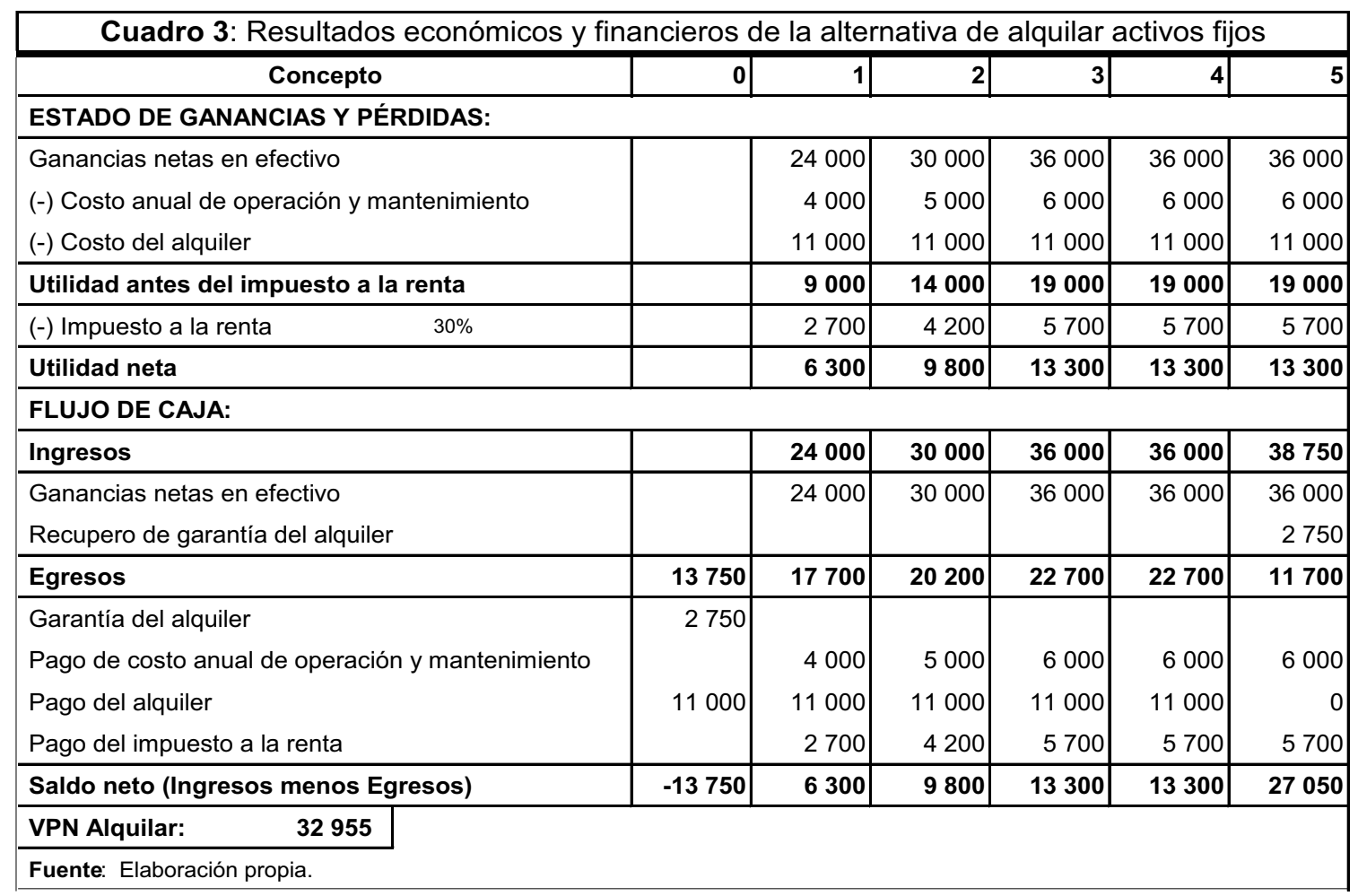

Bajo las premisas planteadas, la alternativa Comprar resulta más atractiva financieramente que $\mathrm{Al}$ quilar, dado que a un costo de oportunidad de $12 \%$ anual, el VPN de Comparar es mayor que el VPN de Alquilar, pero con una diferencia en el VPN que no es significativa. Si la empresa optase por Alquilar -en lugar de la mejor decisión que es Comprar-se obtendría una menor ganancia ( $\mathrm{S} / .1103)$, monto que representa una variación de $3,2 \%$ con respecto al valor de la alternativa óptima.

Una manera de verificar si la diferencia mostrada entre el VPN de alquilar y el VPN de comprar es significativa, es sensibilizar el VPN ante cambios en los valores utilizados como premisas del modelo financiero. Las variaciones que se pueden analizar son el monto de la inversión, el costo y la garantía exigida por el alquiler, así como la tasa correspondiente al costo de oportunidad de los accionistas de la empresa.

Paralelamente, en nuestra aplicación, se puede apreciar que la disponibilidad de caja de la alternativa de comprar es atractiva en todos los años del horizonte planeado, en comparación con la alternativa de alquilar. De esta manera, es posible mejorar el valor de los resultados de la alternativa de comprar, financiando parte de la inversión en activos fijos con deuda en condiciones competitivas, lo cual depende de la situación integral de la empresa y de sus proyecciones, así como de la disposición de las entidades financieras de apoyar con créditos a la empresa objeto del análisis.

\section{MODELO FINANCIERO DE COSTOS DIFERENCIALES}

Ahora bien, si revisamos la información de la aplicación que interviene en el análisis de las alternativas de comprar y alquilar de activos fijos, se aprecian valores comunes a ambas alternativas, que en términos comparativos -para seleccionar la mejor alternativa- pueden ser excluidos de las proyecciones. Este tipo de análisis da lugar a un modelo financiero alternativo, el cual consiste en comparar solo los costos diferenciales, es decir, aquellos conceptos que hacen diferente una alternativa de otra. Los valores comunes que no se consideran en el análisis, en la aplicación, son las ganancias netas en efectivo y el costo de operación y mantenimiento de los activos fijos.

Este modelo, denominado modelo financiero de costos diferenciales, considera el flujo de caja de costos (pagos o desembolsos en efectivo) incluyendo el efecto fiscal de la depreciación (caso de comprar) y del costo del alquilar, además de otros rubros que, en el análisis comparativo, pueden resultar en costo o en beneficio, según corresponda. Si los costos se presentan en valor absoluto (positivo), los beneficios deben mostrarse con signo negativo; o viceversa. Bajo la alternativa de comprar, la inversión es un egreso de caja 


\begin{tabular}{|c|c|c|c|c|c|c|}
\hline Concepto & 0 & 1 & 2 & 3 & 4 & 5 \\
\hline \multicolumn{7}{|l|}{ COMPRAR: } \\
\hline Inversión en activos fijos & 50000 & 0 & 0 & 0 & 0 & 0 \\
\hline (-) Ahorro fiscal de la depreciación de activos fijos & & 1875 & 1875 & 1875 & 1875 & 1875 \\
\hline (-) Valor residual de activos fijos & & & & & & 18750 \\
\hline Saldo neto del flujo de caja de costos (comprar) & 50000 & -1875 & -1875 & -1875 & -1875 & -20625 \\
\hline \multicolumn{7}{|l|}{ VPN Costos (Comprar): $\quad 32602$} \\
\hline \multicolumn{7}{|l|}{ ALQUILAR: } \\
\hline Garantía del alquiler & 2750 & 0 & 0 & 0 & 0 & \\
\hline Pago del alquiler & 11000 & 11000 & 11000 & 11000 & 11000 & \\
\hline (-) Recupero de garantía del alquiler & & & & & & 2750 \\
\hline (-) Ahorro fiscal del alquiler & 0 & 3300 & 3300 & 3300 & 3300 & 3300 \\
\hline Saldo neto del flujo de caja de costos (alquilar) & 13750 & 7700 & 7700 & 7700 & 7700 & -6050 \\
\hline VPN Costos (Alquilar): & & & & & & \\
\hline Fuente: Elaboración propia. & & & & & & \\
\hline
\end{tabular}

en el año cero, que no se presenta con la alternativa de Alquilar. El efecto fiscal de la depreciación, la cual no es egreso de caja, se observará por el menor pago del impuesto a la renta (que resulta de aplicar la tasa del impuesto a la renta al monto de la depreciación anual); en cambio, el efecto fiscal del costo de alquilar, el cual además es egreso de caja, implica también un menor pago del impuesto a la renta.

En el Cuadro 4 se muestran los resultados del flujo de caja de costos diferenciales netos para las alternativas de Comprar y Alquilar activos fijos.

Como se puede apreciar en el Cuadro 4, en términos de costos netos, también la alternativa comprar resulta más atractiva que la alternativa de alquilar (por registrar el menor valor del VPN de costos netos), manteniéndose la diferencia entre ambas alternativas en S/.1103 (tal como se observa en el análisis completo de rentabilidad). De esta manera, el criterio aplicado es que al minimizar los costos entre alternativas, bajo el supuesto de iguales ingresos y de otros costos comunes a las alternativas analizadas, se maximiza en valor del VPN.

Naturalmente, el modelo de costos diferenciales netos es una simplificación de mucha ayuda en la toma de decisiones, pero tiene la limitante que al no mostrar todos los componentes de los resultados proyectados del negocio bajo análisis, no facilita apreciar otros aspectos de la empresa, como por ejemplo, la disponibilidad de caja para asumir obligaciones que permitan financiar la compra o el alquiler de los activos fijos.

A continuación se realiza una prueba de sensibilidad en el VPN de costos diferenciales netos, para las alternativas de comprar y alquilar, ante cambios en los valores de la inversión y el costo del alquiler, incluyendo en cada caso diversos niveles del costo de oportunidad del accionista. Para determinar el punto de indiferencia entre comprar y alquilar se utilizará el VPN del flujo marginal (VPN Comprar-Alquilar), es decir, el VPN del flujo de costos netos de Comprar menos el flujo de costos de la alternativa de Alquilar.

El flujo neto de caja marginal de costos entre las alternativas analizadas se presenta en el Cuadro 5 , en donde se tiene que para comprar, con respecto a alquilar, se requieren fondos adicionales por S/. 36250 y a cambio se tendrían mayores beneficios netos (en el cuadro en signo negativo por el supuesto considerado anteriormente).

\begin{tabular}{|l|r|r|r|r|r|r|}
\hline \multicolumn{6}{|c|}{ Cuadro 5: Flujo de caja de costos marginales, entre comprar y alquilar activos fijos } \\
\hline \multicolumn{1}{|c|}{ Concepto } & $\mathbf{0}$ & $\mathbf{1}$ & $\mathbf{2}$ & $\mathbf{3}$ & $\mathbf{4}$ & $\mathbf{5}$ \\
\hline Saldo neto del flujo de caja de costos (comprar) & 50000 & -1875 & -1875 & -1875 & -1875 & -20625 \\
Saldo neto del flujo de caja de costos (alquilar) & 13750 & 7700 & 7700 & $\mathbf{7 7 0 0}$ & $\mathbf{7 7 0 0}$ & -6050 \\
\hline Saldo neto diferencial (comprar menos alquilar) & $\mathbf{3 6 ~ 2 5 0}$ & $\mathbf{- 9 5 7 5}$ & $\mathbf{- 9 5 7 5}$ & $\mathbf{- 9 5 7 5}$ & $\mathbf{- 9 5 7 5}$ & $\mathbf{- 1 4 5 7 5}$ \\
\hline VPN Costos (Comprar menos Alquilar): -1 103 & \multicolumn{5}{|l}{} \\
\hline Fuente: Elaboración propia.
\end{tabular}




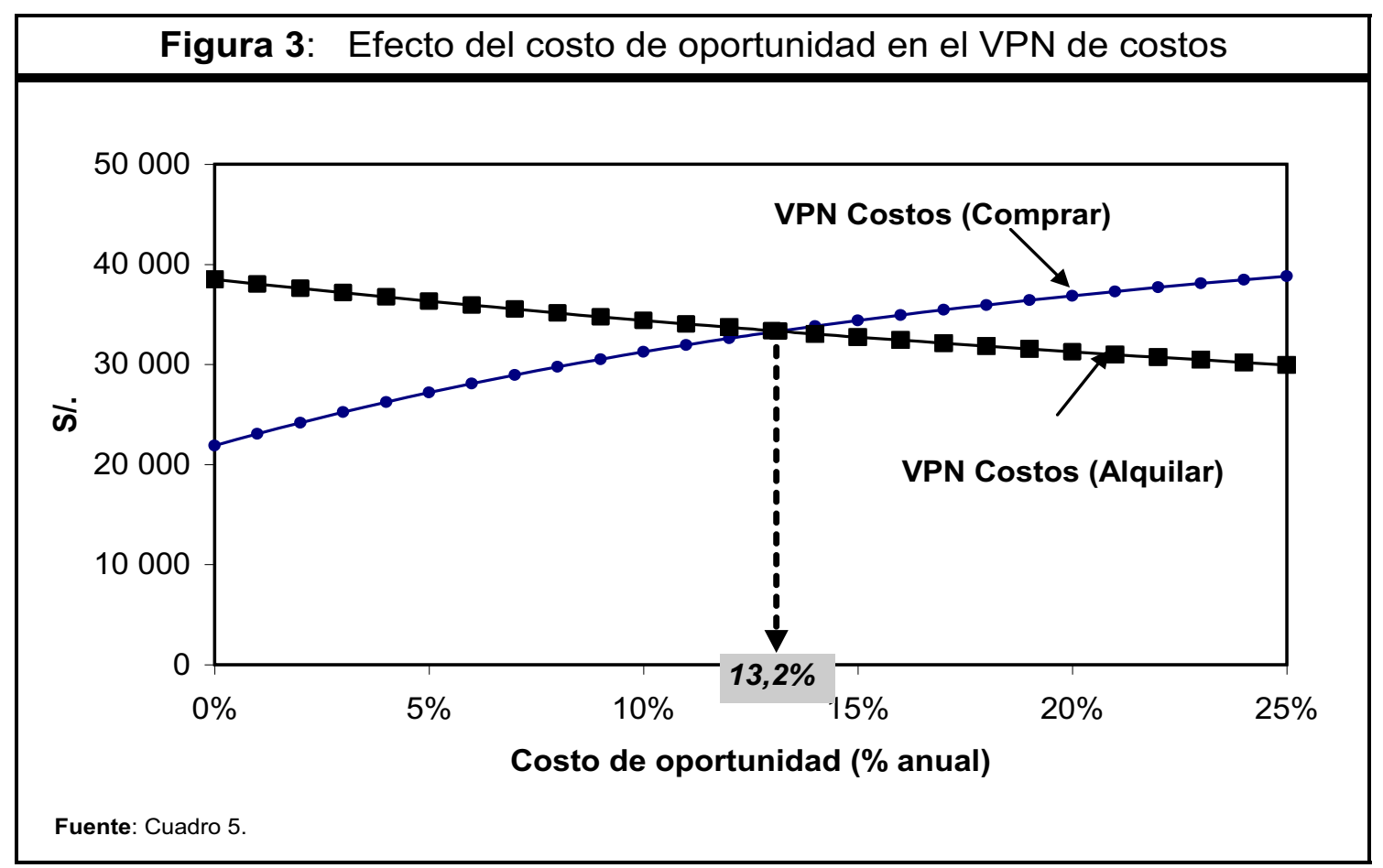

\begin{tabular}{|l|r|r|r|r|r|r|r|r|r|r|}
\hline \multicolumn{2}{|c|}{ Cuadro 6: VPN diferencial (12\% anual) según inversión (comprar) y alquiler de activos fijos } \\
\cline { 2 - 11 } & \multicolumn{7}{|c|}{ Alquiler (S/. / Año) } \\
\hline & 24000 & -2735 & -8864 & -16953 & -18056 & -19588 & -21120 & -22652 & -24184 \\
\cline { 2 - 10 } & 30000 & 1177 & -4951 & -13041 & -14144 & -15676 & -17208 & -18740 & -20272 \\
\cline { 2 - 10 } & 40000 & 7697 & 1569 & -6520 & -7623 & -9155 & -10687 & -12219 & -13751 \\
\cline { 2 - 10 } & $\mathbf{5 0 0 0 0}$ & 14217 & 8089 & 0 & -1103 & -2635 & -4167 & -5699 & -7231 \\
\cline { 2 - 10 } & 51692 & 15321 & 9193 & 1103 & 0 & -1532 & -3064 & -4596 & -6128 \\
\cline { 2 - 10 } & 60000 & 20738 & 14610 & 6521 & 5417 & 3885 & 2353 & 821 & -711 \\
\hline
\end{tabular}

De esta manera se valida que, para un costo de oportunidad de $12 \%$ anual, la alternativa de Comprar genera, en términos del VPN un ahorro neto actualizado (al año cero) de S/.1103, con respecto a la alternativa de Alquilar.

\section{Efecto del costo de oportunidad}

Para la aplicación que se viene analizando, en la figura 3 , se tiene que para un costo de oportunidad de $13,2 \%$ anual, asumiendo constante los otros factores del análisis, ambas alternativas son indiferentes, dado que registran el mismo valor de VPN; es decir el VPN de costos diferencial (comprar menos alquilar) es cero.

Asimismo, en la indicada figura se tiene que para un menor valor del costo de oportunidad a $13,2 \%$ anual (por ejemplo por la percepción de menor riesgo), la alternativa de Comprar resulta más conveniente que Alquilar (por registrar menor VPN). Para un costo de oportunidad mayor a la tasa indicada (por ejemplo por efecto de un mayor riesgo), conviene la alternativa de alquilar que comprar activos fijos.

\section{Efectos de la inversión y del costo de alquilar}

En el Cuadro 6 se observan los valores del VPN diferencial (comprar menos alquilar), para un costo de oportunidad de $12 \%$ anual. Para los valores del escenario base de comprar (inversión por S/. 50 000) y de alquilar (costo anual de S/. 11000 ), la mejor alternativa es comprar, como se explicó anteriormente.

Así se tiene que, para un costo de alquiler de S/.11000 anuales, el punto de indiferencia entre alquilar y comprar se presenta para una inversión de S/.51 692, 
para montos menores de inversión a dicha suma conviene comprar activos fijos y para valores superiores de inversión, resulta mejor la alternativa alquilar. Por su parte, si la inversión fuese un valor fijo (S/. 50 000), la indiferencia entre comprar y alquilar se da para un alquiler de S/.10 640 al año, para mayores montos de alquiler resulta atractivo comprar y para valores menores es conveniente comprar activos fijos.

De esta manera, utilizando un modelo financiero como el estructurado para la aplicación, es posible simular diversos escenarios futuros de decisión, para establecer situaciones de indiferencia entre comprar y alquilar activos fijos, así como condiciones de preferencia entre comprar y alquilar.

Toda vez que las decisiones financieras se basan en escenarios futuros supuestos, es imperativo que la decisión final de la empresa considere otros aspectos, como la disponibilidad de efectivo para afrontar el pago de las obligaciones que se asuman, aspectos de disponibilidad en el mercado de proveedores de activos fijos para la compra y el alquiler de equipos, estrategias de crecimiento de la empresa, entre otros.

\section{CONCLUSIONES}

1. Las empresas requieren adoptar decisiones que contribuyan a mejorar el valor de las acciones representativas de su capital social. Como parte de su estrategia de crecimiento y/o de optimización de costos, deben seleccionar entre comprar y alquilar activos fijos, lo cual tiene implicancias tributarias en los resultados económicos y financieros de la organización.

2. La estructuración de un modelo financiero para el análisis de las alternativas de comprar y alquilar de activos fijos es necesario como un instrumento de apoyo en las decisiones de la empresa. Dicho análisis se puede realizar utilizando el modelo convencional de determinar los criterios de rentabilidad de las alternativas $u$ aplicando el concepto de costos diferenciales netos de las alternativas. Este último modelo, alternativo al modelo financiero convencional, toma en cuenta -al comparar flujo de costoslos beneficios resultantes para la empresa en los aspectos fiscales (impuesto a la renta y participación de trabajadores en las utilidades de ser el caso).

3. Tanto en el modelo financiero convencional (general), como en el modelo financiero alternativo (de costos netos diferenciales), además de las proyecciones económicas y financieras del negocio, según cada alternativa analizada, se requiere un estimado razonable del costo de oportunidad de los accionistas, siendo uno de los principales componentes de dicho costo, el nivel de riesgo que perciben los inversionistas del negocio y del país en que desarrolle la actividad empresarial.

4. Toda vez que las decisiones financieras de comprar y alquilar de activos fijos se basan en el VPN, como criterio de rentabilidad de mediano y largo plazo, es imperativo que las decisiones para ser integrales involucren otros aspectos del quehacer empresarial, sean del medio interno a la organización o de su entorno externo.

\section{REFERENCIAS BIBLIOGRÁFICAS}

1. Blank, L. y Tarquin, A. (2004). Ingeniería Económica. Quinta edición, McGraw-Hill Interamericana Editores S.A. de C.V., México.

2. Comisión Nacional Supervisora de Empresas y Valores - CONASEV (2008). Información financiera de las empresas listadas en la Bolsa de Valores de Lima. http://www.sbs.gob.pe/ portalsbs/tipotasa/indice.htm (visitado el 200812-02).

3. Fernández, P. (2002). Valoración de empresas. Segunda edición, Ediciones Gestión 2000, España.

4. Moyer, R. Ch., McGuigan, J.R., Kretlow, W.J. (1998). Administración financiera contemporánea. Séptima edición, International Thomson Editores, México.

5. Superintendencia de Banca, Seguros y AFP SBS (2008). Tasas de interés activas en el sistema bancario. http://www.sbs.gob.pe/portalsbs/tipotasa/indice.htm (visitado el 2008-12-12). 